\title{
The role of secondary Reggeons in central meson production
}

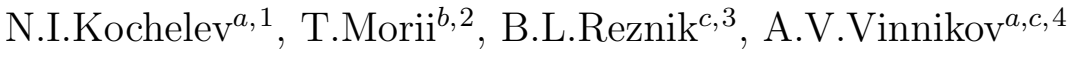 \\ a Bogoliubov Laboratory of Theoretical Physics, \\ Joint Institute for Nuclear Research, \\ Dubna, Moscow region, 141980 Russia \\ ${ }^{b}$ Faculty of Human Development, Division of Sciences for Natural Environment \\ and Graduate School of Science and Technology, \\ Kobe University, Nada, Kobe 657-8501, Japan \\ c Far Eastern State University, Sukhanova 8, GSP, Vladivostok, 690600 Russia
}

\begin{abstract}
We estimate the contribution of $f_{2}$ trajectory exchange to the central $\eta$ and $\eta^{\prime}$ production. It is shown that secondary Reggeons may give a large contribution to processes of double diffractive meson production at high energies.
\end{abstract}

PACS number(s): 12.40.Nn, 13.60.Le, 12.39.Mk

\footnotetext{
${ }^{1}$ e-mail address: kochelev@thsun1.jinr.ru

${ }^{2}$ e-mail address: morii@kobe-u.ac.jp

${ }^{3}$ e-mail address: reznik@dvgu.ru

${ }^{4}$ e-mail address: vinnikov@thsun1.jinr.ru
} 
The Regge theory provides a natural and economical description of hadron-hadron interactions at high energies and small momentum transfers [1]. In this approach the interaction between colliding particles is described by the exchange of effective particles, i.e. Reggeons. At high energies, the pomeron with vacuum quantum numbers gives the dominant contribution to the hadron-hadron total cross sections. The Reggeons with quantum numbers different from the vacuum ones can also contribute to the total cross sections and their contribution is vanishing at very high energies.

An interest in double diffractive processes (DDP) is mediated by the idea that they can be a pure source of glueballs [2], [3]. Intensive studies of these processes have been recently performed by WA102 collaboration at CERN. The mechanism of the central meson production in DDP at high energies is usually related to the double pomeron exchange (DPE) [3], [4], [5]. This conclusion is based on the following observations:

i) if the two-pomeron fusion contributes dominantly to the central meson production, one can explain rather weak energy dependence of the total production cross sections;

ii) $t$ - and azimuthal dependences of differential cross sections for the most mesons are consistent with the two vector-fusion mechanism and with additional assumption that pomeron transforms as vector current;

iii) quantum numbers of these effective vector exchanges, e.g. flavour, P- and C-parities, are the same as for the pomeron.

However, these arguments fail if one looks into the details of the experimental data. For example, even in the simplest case of the light pseudoscalar meson production we find:

i) the observed cross section of $\eta$ production is larger than that of the $\eta^{\prime}$ production [6], while the DPE mechanism predicts the opposite: $\sigma_{\eta}<<\sigma_{\eta^{\prime}}$. This conclusion comes from the consideration of the flavour-singlet structure of the two-pomeron fusion which leads to the enhancement of flavour-singlet meson production [8].

ii) the cross section of $\pi^{0}$ meson production does not show any angular dependence in the range $0^{\circ}<\varphi<150^{\circ}$ and shows a peak (presumably a contribution from the diffractive $\Delta$ and nucleon resonance production [7]) at $\varphi=180^{\circ}$, while the mechanism of two-vector fusion predicts the behaviour like $\sin ^{2} \varphi$ with a maximum at $90^{\circ}$. Hence, the mechanism of $\pi^{0}$ production is not consistent with the two-vector fusion at all.

The main goal of this letter is to underline the importance of secondary Reggeon exchanges for central meson production. As an example, we estimate the contribution of the $f_{2}$ exchange with $P=C=+1$ to the central production of $\eta$ and $\eta^{\prime}$ mesons for WA102 kinematics.

Let us consider a two-Reggeon fusion contribution to central $\eta, \eta^{\prime}$ productions, as is presented in Fig. 1, where the pomeron and $f_{2}$ Reggeon are taken into consideration.

The cross section of meson production in the reaction

$$
p\left(p_{1}\right)+p\left(p_{2}\right) \rightarrow p\left(p_{1}^{\prime}\right)+p\left(p_{2}^{\prime}\right)+M\left(p_{M}\right)
$$

is given by the formula

$$
d \sigma=\frac{d P S^{3}}{4 \sqrt{\left(p_{1} \cdot p_{2}\right)^{2}-m_{p}^{4}}} \sum_{\text {spin }}|T|^{2},
$$

where $d P S^{3}$ is the 3 -body phase space volume, $m_{p}$ is the proton mass, and $T$ is the matrix element for DDP reaction. $\sum_{\text {spin }}$ stands for the summation and averaging over the spins in the final and initial proton states, respectively.

At high energies and small momentum transfers, the four-momenta of initial and final protons in the center-of-mass system are given as follows:

$$
p_{1} \approx\left(P+m_{p}^{2} / 2 P, \overrightarrow{0}, P\right), \quad p_{2} \approx\left(P+m_{p}^{2} / 2 P, \overrightarrow{0},-P\right)
$$




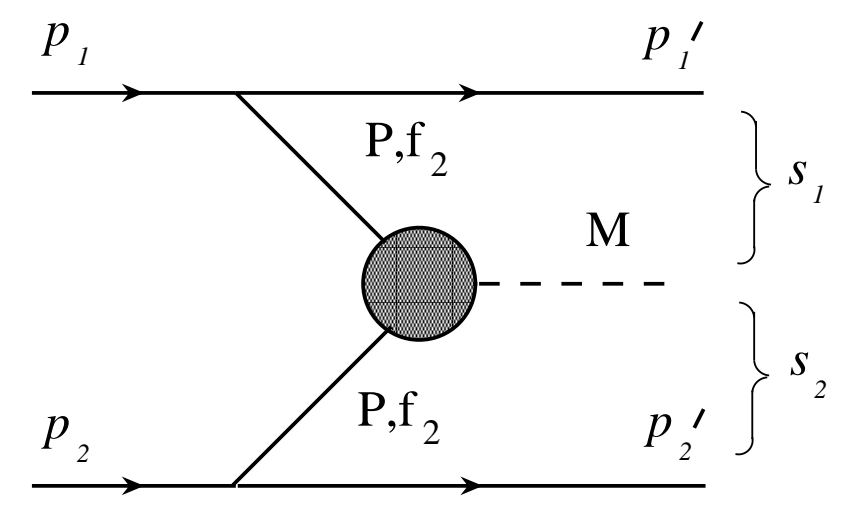

Figure 1: Two Reggeon fusion diagram

$$
\begin{aligned}
& p_{1}^{\prime} \approx\left(x_{1} P+\left(m_{p}^{2}+\vec{p}_{1 T}^{2}\right) / 2 x_{1} P, \vec{p}_{1 T}, x_{1} P\right) \\
& \left.p_{2}{ }^{\prime} \approx\left(x_{2} P+\vec{p}_{2 T}^{2}\right) / 2 x_{2} P, \vec{p}_{2 T},-x_{2} P\right)
\end{aligned}
$$

where $P=\sqrt{s} / 2, s=\left(p_{1}+p_{2}\right)^{2}$. Using the result of Ref. [9] for the high energy phase space at small momentum transfers, we obtain

$$
d P S^{3}=\frac{1}{2^{8} \pi^{4}} d t_{1} d t_{2} d x_{1} d x_{2} d \Phi \delta\left(s\left(1-x_{1}\right)\left(1-x_{2}\right)-M^{2}\right)
$$

for the phase space volume in the DDP reaction, where $\Phi$ is azimuthal angle between final protons, $t_{1,2}=\left(p_{1,2}-p_{1,2}^{\prime}\right)^{2}$ and $M$ is the meson mass. Kinematic limits for the phase space integration in (16) are determined by positive $\vec{p}_{1,2 T}^{2}$ in (15) and the condition $s_{1,2} \geq\left(M+m_{p}\right)^{2}$, where

$$
s_{1,2}=s\left(1-x_{1,2}\right)+m_{p}^{2}+2 t_{1,2} .
$$

Let us consider typical values of $s_{1,2}$ for the diffractive process, which appeared to be very important to understand the reaction mechanism. In the diffractive region where $t_{1,2}$ are small $s_{1,2}$ at given $s$ can be functions of $x_{1,2}$ only. One can see from (4) that $x_{1,2}$ are not independent variables. At fixed $x_{F}=x_{2}-x_{1}$

$$
x_{1}=1-\sqrt{\frac{x_{F}^{2}}{4}+\frac{M^{2}}{s}}-\frac{x_{F}}{2}, \quad x_{2}=1-\sqrt{\frac{x_{F}^{2}}{4}+\frac{M^{2}}{s}}+\frac{x_{F}}{2} .
$$

Using (5) and (6) we obtain the dependence of $s_{1,2}$ on $x_{F}$ (see Fig. 2). As one can see, despite the large value of the total invariant mass of the system, the value of $s_{2}$ is always rather small. Therefore, the large contribution from secondary Regge trajectories can be expected for any central meson production. Fixing $x_{F}$ and varying $s$ (see Fig. 3), we see that this conclusion remains valid even at very high energies. This is why even at LHC energy the contribution from secondary Reggeon exchanges can be not small, contrary to the expectation [11].

Let us estimate more accurately the contribution of both pomeron and secondary Reggeons to the central $\eta, \eta^{\prime}$ production. The leading correction to the two-pomeron fusion contribution to this reaction comes from the additional pomeron- $f_{2}$ Reggeon fusion, where $f_{2}$ is the trajectory with $\mathrm{P}=\mathrm{C}=+1$ and positive signature.

The matrix element of DPE reaction, $T$, is given by the formula:

$$
T=i 36 \beta_{P}^{2} \lambda_{M} A_{P P}^{M} \epsilon_{\mu \nu \rho \sigma} p_{1}^{\mu} p_{2}^{\nu} p_{1}^{\prime \rho} p_{2}^{\prime \sigma} F_{p}\left(t_{1}\right) F_{p}\left(t_{2}\right) F_{P P M}\left(t_{1}, t_{2}\right)
$$




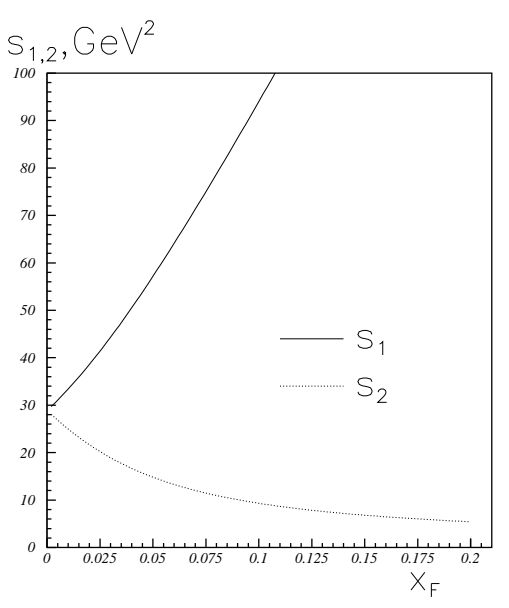

Figure 2: The dependence of the $s_{1,2}$ on $x_{F}$ for $\eta^{\prime}$ production at $P$ $=450 \mathrm{GeV} / \mathrm{c}$.

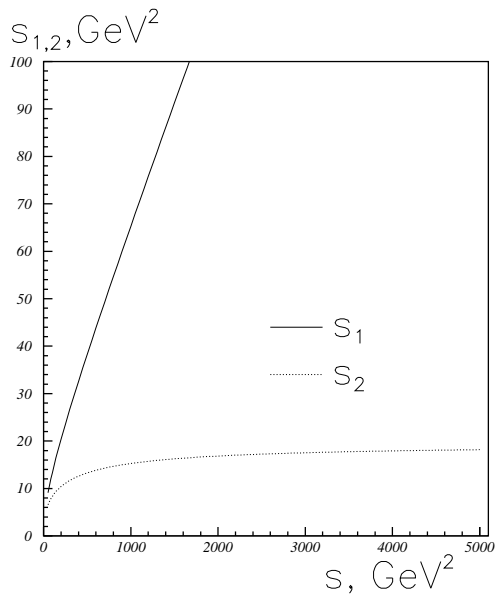

Figure 3: The dependence of the $s_{1,2}$ on $s$ for $\eta^{\prime}$ production at $x_{F}$ $=0.05$.

where

$$
F_{p}=\frac{4 m_{p}^{2}-2.79 t}{\left(4 m_{p}^{2}-t\right)(1-t / 0.71)^{2}}
$$

and

$$
F_{P P M}\left(t_{1}, t_{2}\right)=\frac{1}{\left(1-t_{1} / 8 \pi^{2} f_{P S}^{2}\right)\left(1-t_{2} / 8 \pi^{2} f_{P S}^{2}\right)}
$$

are form factors in proton-pomeron and pomeron-pomeron-pseudoscalar vertices, respectively (see [8]), and

$$
A_{P P}^{M}=\beta_{P}^{4} D_{P P}^{M}\left(\frac{s_{1}}{s_{0}}\right)^{\alpha_{P}\left(t_{1}\right)-1}\left(\frac{s_{2}}{s_{0}}\right)^{\alpha_{P}\left(t_{2}\right)-1} \exp \left(-\frac{i \pi}{2}\left[\alpha_{P}\left(t_{1}\right)+\alpha_{P}\left(t_{2}\right)-2\right]\right),
$$

$s_{0}=1 \mathrm{GeV}^{2}, \beta_{P}=1.8 \mathrm{GeV}^{-1}, \alpha_{P}(t)=1+\epsilon+\alpha^{\prime} t$ is the pomeron trajectory with $\epsilon \approx 0.08, \alpha^{\prime} \approx$ $0.25 \mathrm{GeV}^{-2}$ and

$$
\lambda_{M}=\frac{18}{R_{M} \alpha_{e m}} \sqrt{\frac{2 \Gamma_{\gamma \gamma}}{\pi M^{3}}} .
$$

Here $\Gamma_{\eta \rightarrow \gamma \gamma}=0.46 \times 10^{-6} \mathrm{GeV}, \Gamma_{\eta \prime \rightarrow \gamma \gamma}=4.28 \times 10^{-6} \mathrm{GeV}$ and factors $D_{P P}^{M}$ and $R^{M}$ are related to the wave functions of $\eta$ and $\eta$ '

$$
\begin{gathered}
\eta=-\sin \Theta \eta_{0}+\cos \Theta \eta_{8}, \quad \eta^{\prime}=\cos \Theta \eta_{0}+\sin \Theta \eta_{8}, \\
D_{P P}^{\eta}=-\sin \Theta, \quad D_{P P}^{\eta^{\prime}}=\cos \Theta \\
R_{\eta}=2 \sqrt{2} \cos \Theta-\sin \Theta, \quad R_{\eta^{\prime}}=2 \sqrt{2} \cos \Theta+\sin \Theta,
\end{gathered}
$$

where $\Theta=-19.5^{\circ}$ is the singlet-octet mixing angle.

Using (4), (6), (7) and the equations

$$
\vec{p}_{1,2 T}^{2}=-x_{1,2} t_{1,2}-\left(1-x_{1}\right)^{2} m_{p}^{2}
$$

which follow from (3), the cross section is finally written as

$$
\begin{array}{r}
\frac{d \sigma}{d t_{1} d t_{2} d x_{F} d \Phi}=\frac{3^{4} \lambda_{M}^{2} F_{p}^{2}\left(t_{1}\right) F_{p}^{2}\left(t_{2}\right) F_{P P M}^{2}\left(t_{1}, t_{2}\right)}{2^{9} \pi^{4} \sqrt{x_{F}^{2}+4 M^{2} / s}} \\
\times\left(x_{1} t_{1}+\left(1-x_{1}\right)^{2} m_{p}^{2}\right)\left(x_{2} t_{2}+\left(1-x_{2}\right)^{2} m_{p}^{2}\right)\left|A_{P P}^{M}\right|^{2} \sin ^{2} \Phi .
\end{array}
$$


The $f_{2}$ Reggeon gives an additional contribution to the total amplitude

$$
A^{M}=A_{P P}^{M}+A_{P f_{2}}^{M}+A_{f_{2} P}^{M},
$$

where

$$
\begin{aligned}
& A_{P f_{2}}^{M}=\beta_{P}^{2} \beta_{f_{2}}^{2} D_{P f_{2}}^{M}\left(\frac{s_{1}}{s_{0}}\right)^{\alpha_{P}\left(t_{1}\right)-1}\left(\frac{s_{2}}{s_{0}}\right)^{\alpha_{f_{2}}\left(t_{2}\right)-1} \exp \left(-\frac{i \pi}{2}\left[\alpha_{P}\left(t_{1}\right)+\alpha_{f_{2}}\left(t_{2}\right)-2\right]\right), \\
& A_{f_{2} P}^{M}=\beta_{P}^{2} \beta_{f_{2}}^{2} D_{P f_{2}}^{M}\left(\frac{s_{1}}{s_{0}}\right)^{\alpha_{f_{2}}\left(t_{1}\right)-1}\left(\frac{s_{2}}{s_{0}}\right)^{\alpha_{P}\left(t_{2}\right)-1} \exp \left(-\frac{i \pi}{2}\left[\alpha_{f_{2}}\left(t_{1}\right)+\alpha_{P}\left(t_{2}\right)-2\right]\right) .
\end{aligned}
$$

The factors $D_{i j}^{M}$ can be obtained from the quark decomposition of $\eta_{1}, \eta_{8}$ and $f_{2}$ mesons,

$$
\begin{array}{r}
\eta_{1}=\frac{1}{\sqrt{3}}(u \bar{u}+d \bar{d}+s \bar{s}), \\
\eta_{8}=\frac{1}{\sqrt{6}}(u \bar{u}+d \bar{d}-2 s \bar{s}), \\
f_{2}=\frac{1}{\sqrt{2}}(u \bar{u}+d \bar{d}),
\end{array}
$$

where we assume that $f_{2}$ is an ideal mixing of $S U(3)$ flavour octet and singlet.

We have

$$
D_{P f_{2}}^{\eta}=-\sqrt{\frac{2}{3}} \sin \Theta+\frac{1}{\sqrt{3}} \cos \Theta, \quad D_{P f_{2}}^{\eta^{\prime}}=\sqrt{\frac{2}{3}} \cos \Theta+\frac{1}{\sqrt{3}} \sin \Theta .
$$

The parameters of $f_{2}$ trajectory have been taken from Donnachie-Landshoff fit [10]

$$
\beta_{f_{2}}=3.6 \mathrm{GeV}^{-1}, \quad \epsilon_{f_{2}}=-0.45,
$$

with $\alpha_{f_{2}}^{\prime} \approx 0.9 \mathrm{GeV}^{-2}$.

The final result for $\eta$ and $\eta$ ' production cross sections for WA102 kinematics $(P=450 \mathrm{GeV} / \mathrm{c}$, $\left.0<x_{F}<0.1\right)$ becomes to be equal to

$$
\sigma(\eta)=450 \mathrm{nb}, \quad \sigma\left(\eta^{\prime}\right)=550 \mathrm{nb} .
$$

which can be compared with the DPE contribution alone [8]:

$$
\sigma(\eta)=46 \mathrm{nb}, \quad \sigma(\eta \prime)=370 \mathrm{nb},
$$

and with the experimental data [6]

$$
\sigma(\eta)=1295 \pm 120 \mathrm{nb}, \quad \sigma\left(\eta^{\prime}\right)=588 \pm 60 \mathrm{nb} .
$$

We see that $f_{2}$ Reggeon contribution to the cross section of $\eta$ meson production is very large. The admixture of flavour non-singlet $f_{2}$ exchange increases the cross section by an order of magnitude and also gives the large enhancement of $\eta^{\prime}$ production. Taking into account uncertainties of our model for possible values of quark-pomeron and quark- $f_{2}$ coupling constants as well as representation of form factors in effective vertices, we conclude that the sum of DPE and $f_{2}$ exchange can explain the observed value of total cross section of $\eta$ and $\eta \prime$ central production. On the other hand, the DPE alone fails to explain the cross section value. We should stress that $f_{2}$ exchange does not spoil a good DPE description of the $t$ and azimuthal dependence of the 


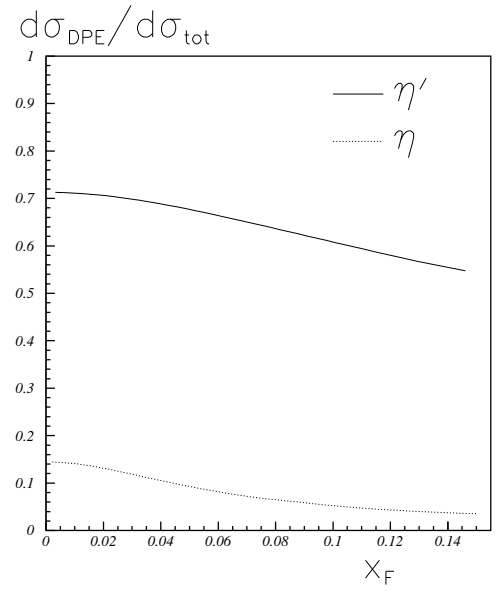

Figure 4: $d \sigma_{D P E} / d \sigma_{t o t}$ as a function of $x_{F}$.

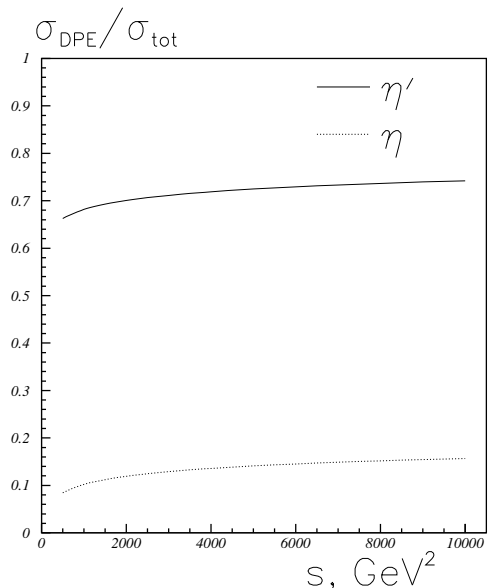

Figure 5: $\sigma_{D P E} / \sigma_{t o t}$ as a function of $s$.

differential cross sections of $\eta$ and $\eta^{\prime}$ production [8], which is just fixed by the shape of nucleon and pseudoscalar meson form factors and vector-like structure of quark-Reggeon vertex.

We have also analysed the DPE contribution to the cross section for different values of $x_{F}$ and $s$ (see Figs. 4 and 5). As is seen here, even for $\eta^{\prime}$ production, its contribution is not dominant at any value of $x_{F}$ and $s$. Increasing energy does not lead to the prevailing of DPE in $\eta$ and $\eta \prime$ production. This is the reason why meson production within DDP does not seem to be a pure kinematical region for the dominance of DPE mechanism even at LHC energy [11].

Although our calculation has been performed only for the simplest case of pseudoscalar $\eta$ and $\eta^{\prime}$ meson production, we think that any DDP cannot leave the region where exchanges by secondary Regge trajectories are significant. This conclusion is based mainly on kinematical arguments; this is why we expect them to be correct for any central meson production too. We should also mention that due to large admixture of flavour singlet component, $f_{2}$ exchange can play an important role even in reactions of central glueball production.

Concluding, we have estimated the contribution of secondary Reggeon trajectories into central production of $\eta$ and $\eta^{\prime}$ mesons. The contribution is shown to be very large. Therefore, before to make some definite conclusions about properties of the pomeron from the analysis of central production data, one should carefully disentangle the secondary Reggeon contribution.

We are grateful to A. Dorokhov, S. Gerasimov, V. Romanovsky, N. Russakovich and A. Titov for useful discussions

\section{References}

[1] P.D.B.Collins, An introduction to Regge theory and high energy physics, Cambridge University press, 1977.

[2] D.Robson, Nucl.Phys.B130 (1977) 328.

[3] F.E.Close, Rep.Prog.Phys.51 (1998) 833.

[4] F.E.Close, A.Kirk, G.Schuler, Phys.Lett.B477 (2000) 13.

[5] F.E.Close, G.Schuler, Phys.Lett.B458 (1999) 127.

[6] D.Barberis et al., Phys.Lett.B427 (1998) 398. 
[7] A.Kirk, V.Romanovsky, private communication.

[8] N.I.Kochelev, T.Morii, A.Vinnikov, Phys.Lett.B457 (1999) 202.

[9] S.Frixione, M.L.Mangano, P.Nason and G.Ridolfi, Phys.Lett.B319 (1993) 339.

[10] Donnachie, P.V. Landshoff, Phys.Lett.B296 (1992) 227.

[11] A. Kirk, "A study of Double Pomeron Exchange in ALICE", hep-ph/9811230.

[12] F.E.Close, G.A.Schuler, Phys.Lett.B464 (1999) 279.

[13] D.Barberis et al., Phys.Lett.B467 (1999) 165. 